OPEN ACCESS

Edited by:

Michaela Tencerova, Academy of Sciences of the Czech Republic (ASCR), Czechia

Reviewed by:

Divya Singh

Central Drug Research Institute (CSIR),

India

Mattabhorn Phimphilai,

Chiang Mai University, Thailand Bin Feng Cheng,

Xinxiang Medical University, China

${ }^{*}$ Correspondence:

Yikai Li

ortho@smu.edu.cn

Specialty section: This article was submitted to

Bone Research,

a section of the journa

Frontiers in Endocrinology

Received: 01 December 2021

Accepted: 18 January 2022

Published: 22 February 2022

Citation:

Zheng S, Zhou C, Yang H, Li J, Feng Z, Liao L and Li Y (2022) MelatoninAccelerates Osteoporotic Bone Defect Repair by Promoting Osteogenesis-Angiogenesis Coupling.

Front. Endocrinol. 13:826660. doi: 10.3389/fendo.2022.826660

\section{Melatonin Accelerates Osteoporotic Bone Defect Repair by Promoting Osteogenesis-Angiogenesis Coupling}

\author{
Sheng Zheng ${ }^{1}$, Chunhao Zhou ${ }^{2}$, Han Yang ${ }^{1}$, Junhua Li $^{1}$, Ziyu Feng ${ }^{1}$, Liqing Liao ${ }^{1}$ \\ and Yikai $L^{1 *}$
}

1 School of Traditional Chinese Medicine, Southern Medical University, Guangzhou, China, ${ }^{2}$ Department of OrthopedicsSpine Surgery, Nanfang Hospital, Southern Medical University, Guangzhou, China

Previous studies have revealed that melatonin could play a role in anti-osteoporosis and promoting osteogenesis. However, the effects of melatonin treatment on osteoporotic bone defect and the mechanism underlying the effects of melatonin on angiogenesis are still unclear. Our study was aimed to investigate the potential effects of melatonin on angiogenesis and osteoporotic bone defect. Bone marrow mesenchymal stem cells (BMSCs) were isolated from the femur and tibia of rats. The BMSC osteogenic ability was assessed using alkaline phosphatase (ALP) staining, alizarin red S staining, qRT-PCR, western blot, and immunofluorescence. BMSC-mediated angiogenic potentials were determined using qRT-PCR, western blot, enzyme-linked immunosorbent assay, immunofluorescence, scratch wound assay, transwell migration assay, and tube formation assay. Ovariectomized (OVX) rats with tibia defect were used to establish an osteoporotic bone defect model and then treated with melatonin. The effects of melatonin treatment on osteoporotic bone defect in OVX rats were analyzed using micro-CT, histology, sequential fluorescent labeling, and biomechanical test. Our study showed that melatonin promoted both osteogenesis and angiogenesis in vitro. BMSCs treated with melatonin indicated higher expression levels of osteogenesis-related markers [ALP, osteocalcin (OCN), runt-related transcription factor 2, and osterix] and angiogenesisrelated markers [vascular endothelial growth factor (VEGF), angiopoietin-2, and angiopoietin-4] compared to the untreated group. Significantly, melatonin was not able to facilitate human umbilical vein endothelial cell angiogenesis directly, but it possessed the ability to promote BMSC-mediated angiogenesis by upregulating the VEGF levels. In addition, we further found that melatonin treatment increased bone mineralization and formation around the tibia defect in OVX rats compared with the control group. Immunohistochemical staining indicated higher expression levels of osteogenesisrelated marker (OCN) and angiogenesis-related markers (VEGF and CD31) in the melatonin-treated OVX rats. Then, it showed that melatonin treatment also increased the bone strength of tibia defect in OVX rats, with increased ultimate load and stiffness, as performed by three-point bending test. In conclusion, our study demonstrated that melatonin could promote BMSC-mediated angiogenesis and promote osteogenesisangiogenesis coupling. We further found that melatonin could accelerate osteoporotic 
bone repair by promoting osteogenesis and angiogenesis in OVX rats. These findings may provide evidence for the potential application of melatonin in osteoporotic bone defect.

Keywords: melatonin, osteoporosis, bone defect repair, osteogenesis-angiogenesis coupling, bone marrow mesenchymal stem cells

\section{INTRODUCTION}

Osteoporosis, as the most frequent bone disease, results in reduced bone strength. The main characteristics include lower bone mineral density (BMD) and bone mass, impaired bone quality, and abnormal micro-architecture (1-3). Osteoporosis is a common and age-related bone disease throughout the world, affecting more than 20 million individuals (4), which causes bone fragility and fractures (2). It has a major influence on individuals associated with high morbidity and mortality (5). As a global health concern, osteoporosis can affect both sexes and all races, dramatically increasing the social and economic burden worldwide.

Osteoporosis has been recognized as an increased risk of bone fracture and bone defect healing. Rodent studies showed that osteoporosis could cause a striking reduction in the callus size of bone fracture and bone defect, BMD, and mechanical strength (6). Previous studies showed that the healing time of bone fractures or bone defects was significantly longer in patients with osteoporosis than in healthy people (7-10). Maintaining osteogenesis and angiogenesis is crucial for osteoporotic bone regeneration. Ding et al. found that reduced local blood supply to the tibial metaphysis may be associated with ovariectomyinduced osteoporosis (11). In a rat osteoporotic model, new bone trabeculae is arranged in an irregular and loose fashion, indicating the poor bone quality of newly formed bone (12). For patients with osteoporotic bone defect, osteoclast activity is enhanced and bone resorption proceeds at a faster rate than that of bone formation. In addition, the ability of new bone formation is decreased, and bone defect healing is significantly delayed compared with normal bone defect (13). Thus, the treatment is more difficult than that of normal bone defect. In the face of such a severe health problem, how to find more therapeutic strategies and ideal drugs has become an urgent problem to be solved.

Melatonin, synthesized from serotonin in the pineal gland, is a signal molecule that modulates the biological circadian rhythms in humans (14). Except for the pineal gland, melatonin can also be synthesized locally in the bone marrow. Increasing evidence demonstrates that melatonin may play a critical role in bone metabolism. Melatonin, the synthesis of which decreases with aging, is considered to be involved in age-related bone loss and osteoporosis $(15,16)$. In bone, two types of membrane-bound melatonin receptors, including MT1 and MT2, have been identified and can be expressed in both osteoblasts and osteoclasts (17). The level of melatonin in the bone marrow was twice that of plasma at night (18), suggesting that it may be related to bone metabolism. Multiple studies have revealed that melatonin could promote osteoblast proliferation and differentiation, inhibit osteoclast activity, maintain the steady- state of bone metabolism, and thus play a role in anti-osteoporosis (19-22). Zhang et al. demonstrated that melatonin could restore the osteoporosis-impaired osteogenic potential of bone marrow mesenchymal stem cells (BMSCs) and alleviate bone loss through the HGF/PTEN/Wnt/beta-catenin axis (23). Dong et al. showed that melatonin treatment could upregulate the expressions of neuropeptide $\mathrm{Y}$ and its receptor Y1 and promote mesenchymal stem cell proliferation and migration (24). Thus, it indicates that melatonin may be a potential biomolecule for osteoporosis and its related bone defect.

Moderate osteogenesis and angiogenesis is involved in both bone repair and fracture healing (25). Currently, accumulating evidence has indicated the associations between melatonin and osteogenesis. However, few studies have been conducted to research the relationship between melatonin and angiogenesis. Ramírez-Fernández et al. observed that only the melatonin group showed a significantly increased number of blood vessels compared to the control group in a bone defect rabbit model (26). However, the mechanism underlying the effects of melatonin on angiogenesis was not clarified. Thus, how melatonin affects angiogenesis and what its effects are on osteoporotic bone defect are still unclear. The purpose of this study was to evaluate the potential effects of melatonin on angiogenesis and osteoporotic bone defect, which may provide evidence for the potential application of melatonin in osteoporosis and osteoporotic bone defect.

\section{MATERIALS AND METHODS}

\section{Isolation and Culture of Rat BMSCs}

BMSC isolation was performed as previously described $(27,28)$. BMSCs were harvested from the bone marrow of femurs and tibias in 2-week-old Sprague-Dawley (SD) rats. The rats were euthanized and sterilized in $75 \%$ ethanol for $15 \mathrm{~min}$. BMSCs were flushed out by an injection of alpha modified Eagle's minimum essential medium ( $\alpha$-MEM; HyClone, USA) using a 5 -ml syringe fitted with a 25-gauge needle under sterile conditions. After centrifugation, the BMSCs were cultured in $\alpha$-MEM, which was supplemented with $10 \%$ fetal bovine serum (Gibco, USA) and 1\% penicillin/streptomycin (Gibco, USA). The BMSCs between passages 3 and 5 were used in the following experiments. All of the above-mentioned cells were cultured at $37^{\circ} \mathrm{C}$ in a humidified atmosphere containing $5 \% \mathrm{CO}_{2}$.

\section{Cell Proliferation Assay}

BMSC proliferation was detected using Cell Counting kit- 8 (CCK-8; Dojindo, Kumamoto, Japan) following the manufacturer's instructions. Specifically, the BMSCs were seeded with a density of 2,000 cells per well in a 96-well plate 
and cultured in complete medium containing melatonin with various gradient concentrations $(10 \mathrm{nM}, 100 \mathrm{nM}, 1 \mu \mathrm{M}$, and 10 $\mu \mathrm{M})$ for various durations (1, 3, 5, and 7 days). Melatonin (purity $>99 \%$, cat. no. S20287) was purchased from Yuanye BioTechnology Co., Ltd. (Shanghai, China). The untreated wells served as the control group. Then, each well was subjected to a $10-\mu \mathrm{l}$ CCK- 8 solution, and the cells were incubated at $37^{\circ} \mathrm{C}$ for $1 \mathrm{~h}$. Then, the optical density was measured at $450 \mathrm{~nm}$ using a microplate reader (Thermo, USA).

\section{Osteoblastic Determination and Mineralization Assessment}

BMSCs were seeded in a 24-well plate with a density of $2 \times 10^{4}$ cells per well. The medium was replaced with an osteogenic medium (complete $\alpha$-MEM containing $10 \mathrm{nM}$ dexamethasone, $50 \mu \mathrm{M}$ ascorbic acid, and $10 \mathrm{mM} \beta$-glycerophosphate) after reaching over $80 \%$ confluence. For treatment, melatonin with various gradient concentrations $(10 \mathrm{nM}, 100 \mathrm{nM}$, and $1 \mu \mathrm{M})$ was added into the medium. The untreated wells served as the control group. Then, protein was extracted, and the supernatant liquid was harvested after 7 days of osteogenic induction for western blotting analysis and enzyme-linked immunosorbent assay (ELISA) test of vascular endothelial growth factor (VEGF), respectively. Alkaline phosphatase (ALP) activity was determined at day 7 of differentiation using ALP Staining Kit (cat. no. P0321S, Beyotime Biotechnology, China). The mineralization of the calcium nodule was detected on the 14th day using alizarin red S (ARS) solution (cat. no. G1452, Solarbio Science \& Technology, China). The absorbance at $405 \mathrm{~nm}$ for ALP and $560 \mathrm{~nm}$ for ARS staining was detected using a microplate reader (Thermo, USA).

\section{Quantitative Real-Time PCR}

Prior to PCR, total RNA was extracted using RNA Purification Kit (EZBioscience, USA). The RNA was reverse-transcribed by $500 \mathrm{ng}$ of total RNA from each sample using Reverse Transcription Kit (EZBioscience, USA). Next, the cDNA was amplified with SYBR Green qPCR Master Mix (EZBioscience, USA). Data were analyzed, and the relative expression levels were calculated by the $2^{-\Delta \Delta C T}$ method. Housekeeping gene glyceraldehyde-3-phosphate dehydrogenase (GAPDH) was used for normalization. All reactions were carried out with three biological replicates, and each analysis consisted of three technical replicates. The primer sequences were designed by Oligo 7.0 software and are shown in Table 1.

\section{Western Blotting}

Total protein was extracted by RIPA buffer (Beyotime Biotechnology, China), containing protease and phosphatase inhibitors (Sigma-Aldrich, USA), for $30 \mathrm{~min}$ at $4^{\circ} \mathrm{C}$. The cell lysates were cleared by centrifugation, and the protein concentration was determined using the bicinchoninic acid quantification kit (Beyotime Biotechnology, China). Furthermore, $30 \mu \mathrm{g}$ protein was electrophoresed with $10 \%$ SDS-PAGE electrophoresis (Beyotime Biotechnology, China) and subsequently transferred to a polyvinylidene difluoride membrane (Millipore, USA). The membranes were blocked with $5 \%$ bovine serum albumin (BSA) (Solarbio Science \& Technology, China) for $1 \mathrm{~h}$ at room temperature and incubated overnight at $4^{\circ} \mathrm{C}$ with primary antibodies against ALP (1:1,000; DF6225, Affinity Biosciences, Cincinnati, OH, USA), osteocalcin (OCN) (1:1,000; DF12303, Affinity Biosciences, Cincinnati, OH, USA), runt-related transcription factor 2 (RUNX2) (1:2,000; AF5186, Affinity Biosciences, Cincinnati, OH, USA), VEGF (1:1,000; AF5131, Affinity Biosciences, Cincinnati, OH, USA), GAPDH (1:5,000; T0004, Affinity Biosciences, Cincinnati, OH, USA), and $\beta$-actin (1:5,000; T0022, Affinity Biosciences, Cincinnati, OH, USA). The membranes were then incubated with secondary antibody (1:5,000; S0001, Affinity Biosciences, Cincinnati, USA). Finally, the membranes were visualized with enhanced chemiluminescence reagent (Beyotime Biotechnology, China). The band intensity was quantified using Image Lab (Bio-Rad, Hercules, CA, USA).

\section{VEGF Analysis by ELISA}

Commercial ELISA kit for VEGF (Cusabio, Wuhan, China) was used to determine the concentrations of VEGF in the supernatant liquid from different groups following the manufacturer's protocols.

\section{Immunofluorescence}

BMSCs were fixed with 4\% PFA and permeabilized with $0.1 \%$ Triton X-100 in phosphate-buffered saline (PBS) containing 5\% BSA. After blocking with 5\% BSA for $1 \mathrm{~h}$, the cells were stained overnight at $4^{\circ} \mathrm{C}$ with primary antibodies. Subsequently, the cells

TABLE 1 | Real-time PCR primer sequences used in the study.

\begin{tabular}{|c|c|c|}
\hline Gene & Forward primer $\left(5^{\prime}-3^{\prime}\right)$ & Reverse primer $\left(5^{\prime}-3^{\prime}\right)$ \\
\hline ALP & CCGCAGGATGTGAACTACT & GGTACTGACGGAAGAAGGG \\
\hline $\mathrm{OCN}$ & CAGACAAGTCCCACACAGCA & CCAGCAGAGTGAGCAGAGAGA \\
\hline RUNX2 & ACTTCCTGTGCTCGGTGCT & GACGGTTATGGTCAAGGTGAA \\
\hline OSX & GGAAAAGGAGGCACAAAGAA & CAGGGGAGAGGAGTCCATT \\
\hline VEGF & CACGACAGAAGGGGAGCAGAAAG & GGCACACAGGACGGCTTGAAG \\
\hline Ang-2 & GAAGAAGGAGATGGTGGAGAT & CGTCTGGTTGAGCAAACTG \\
\hline Ang-4 & GCTCCTCAGGGCACCAAGTTC & CACAGGCGTCAAACCACCAC \\
\hline GAPDH & ATGGCTACAGCAACAGGGT & TTATGGGGTCTGGGATGG \\
\hline
\end{tabular}

ALP, alkaline phosphatase; OCN, osteocalcin; RUNX2, runt-related transcription factor 2; OSX, osterix; VEGF, vascular endothelial growth factor; Ang-2, angiopoietin-2; Ang-4, angiopoietin-4; GAPDH, glyceraldehyde-3-phosphate dehydrogenase. 
were incubated with a fluorescein isothiocyanate-conjugated secondary antibody (1:1,000; ab6717, Abcam, UK) for $1 \mathrm{~h}$ and then stained with 4',6-diamidino-2-phenylindole. The fluorescence signal was captured using a fluorescence microscope (DMi8, Leica, Germany).

\section{Angiogenesis-Related Assays in vitro}

To further assess the angiogenic capability of melatonin, the BMSCs were treated with or without $100 \mathrm{nM}$ melatonin, and the conditioned mediums were harvested after 7 days of osteogenic induction, which were used for the following assays. Subsequently, human umbilical vein endothelial cells (HUVECs) (Procell Life Science \&Technology Company, Wuhan, China) were cultured and treated under different conditions (1): fresh medium (2), fresh medium with $100 \mathrm{nM}$ melatonin (3), conditioned medium from BMSCs without melatonin, and (4) conditioned medium from BMSCs with 100 $\mathrm{nM}$ melatonin. Then, scratch wound assay, transwell migration assay, and tube formation assay were further detected as will be detailed in the following discussion.

For the scratch wound assay, HUVECs were seeded at a density of $2 \times 10^{5} /$ well in a 6 -well plate. The cells were scratched after confluence under an inverted microscope (Nikon; Tokyo, Japan). Then, the cells were cultured in the aforementioned mediums. The wound images were obtained immediately and at $12 \mathrm{~h}$ later. The width of the wounded areas (\%) was calculated as $\left(A_{0}-A_{\mathrm{n}}\right) / \mathrm{A}_{0} \times$ 100 , where $A_{0}$ and $A_{\mathrm{n}}$ represent the initial wound area and the residual wound area at the metering point, respectively.

For the transwell migration assay, HUVECs were suspended and loaded into the top chamber of a transwell plate (Corning, NY, USA). The medium from the treated BMSCs was then added to the chamber. After $12 \mathrm{~h}$, the unmigrated HUVECs in the upper chambers were removed by wiping the top of the membranes. The migrated cells were fixed in $4 \%$ paraformaldehyde, washed with PBS solution, and then stained with $0.5 \%$ crystal violet for $10 \mathrm{~min}$. The cells were imaged and counted under the random fields of the microscope (Nikon; Tokyo, Japan).

For the tube formation assay, HUVECs were seeded into a Matrigel-coated 96-well plate at a density of $5 \times 10^{3} /$ well, Then, the cells were incubated in the aforementioned medium. After incubation for $8 \mathrm{~h}$, HUVEC tube formation was observed under an inverted microscope (Nikon; Tokyo, Japan). The number of tubes was calculated by Image-Pro Plus software.

\section{Animal Experiments in Ovariectomized Rats \\ Ethics Statement}

All experiments were approved by the Animal Care and Ethics Committee of the Southern Medical University (no. SMUL2021003), and the procedures were conducted in accordance with the policies of the Ethics Committee for Animal Research.

\section{Animal Surgery and Treatment}

A total of 84 female specific-pathogen-free SD rats (weight $250 \pm$ 20 g; 12 weeks old; purchased from Zhuhai BesTest Bio-Tech Co., Ltd., Guangdong) were used in this experiment. All the rats were housed at a standard room temperature of $22 \pm 2^{\circ} \mathrm{C}$ and humidity of $55-70 \%$ under a 12-h light/dark cycle with free access to food and water. After adaptation, 78 rats were randomly selected for bilateral ovariectomized (OVX) surgery, and 6 rats received sham surgery as previously described (29). After 3 months, 6 OVX and 6 sham surgery rats were selected for micro-computed tomography (micro-CT) and H\&E staining to confirm the OVX rat model of osteoporosis. Then, 72 OVX rats were randomly selected and anesthetized for the longitudinal approach, which was performed on the medial surface of the proximal end with exposure of the proximal anteromedial metaphysis of the right tibia. Specifically, a standardized drill hole defect (3-mm diameter and 4-mm depth) was used to create a monocortical defect. After surgery, all the rats were randomly divided into three groups: low-dose melatonin treatment group (LMEL group, $n=24$ ), high-dose melatonin treatment group (HMEL group, $n=24$ ), and control group (CON group, $n=24$ ). The LMEL and HMEL group rats were intraperitoneally injected with 10 and $50 \mathrm{mg} / \mathrm{kg} /$ day melatonin daily for 4 weeks, respectively. The $\mathrm{CON}$ group was injected with normal saline under the same conditions. The therapeutic dose of melatonin mentioned above was determined based on previous experiments in which melatonin showed protective effects in an OVX rat model $(30,31)$. The right tibiae in rats were harvested and assigned to micro-CT analysis and histological studies, which were randomly selected from each group ( $n=6 /$ group) at 2 weeks after tibia surgery. All the remaining rats were sacrificed, and the right tibiae were harvested at 4 weeks after treatment. Six tibia specimens were randomly selected from each group for micro-CT analysis and histological studies. The remaining tibia specimens were randomly assigned to fluorescent labeling analysis ( $n=6$ for each) and biomechanical test ( $n=6$ for each).

\section{Micro-CT}

Micro-CT analysis (Model $\mu \mathrm{CT}$ 80, Scanco Medical Inc., Brüttisellen, Switzerland) was first used to confirm the success of the osteoporosis model. We selected the first region of interest (ROI) in the trabecular region of the tibia $(1,500 \mu \mathrm{m}$ in length and approximately $300 \mu \mathrm{m}$ below the proximal epiphyseal plate) and reconstructed it by a computer analysis program. The histomorphometric parameters were considered as BMD, bone volume fraction (BV/TV), and trabecular number (Tb.N).

Bone repair was monitored by micro-CT at 2 and 4 weeks after tibia surgery. The central 2.5-mm-diameter region of the 3$\mathrm{mm}$-diameter defect was defined by drawing a circular contour as the second ROI to evaluate bone regeneration within the defect, which could avoid containing the native bone margins and help obtain a consistent volume of interest. After 3D reconstruction, BMD, BV/TV, Tb.N, and trabecular separation (Tb.Sp) in the ROI region were analyzed. All digitalized data and $3 \mathrm{D}$ images were generated by the built-in software of the micro-CT.

\section{Histology and Immunohistochemical Staining}

For histological and immunohistochemical (IHC) analyses, the samples were decalcified in 10\% EDTA for 4 weeks after microCT imaging. Then, $4-\mu \mathrm{m}$-thick sections were then subjected to 
$\mathrm{H} \& \mathrm{E}$ staining and Masson's trichrome staining. For IHC staining, $6-\mu \mathrm{m}$-thick sections were incubated with primary antibodies against OCN (1:100; DF12303, Affinity Biosciences, Cincinnati, OH, USA), VEGF (1:200; AF5131, Affinity Biosciences, Cincinnati, OH, USA), and CD31(1:200; AF6191, Affinity Biosciences, Cincinnati, OH, USA). The immunoreactivity of the analysis was determined using horseradish peroxidase detection system.

\section{Sequential Fluorescent Labeling}

All of the rats were intraperitoneally injected with $10 \mathrm{mg} / \mathrm{kg}$ calcein (cat. no. C0875, Sigma) at 10 and 3 days before the end of the experiment (32). At the end of the observation time (4 weeks after the treatment), the tibia samples were obtained for hardtissue slicing and then imaged through the laser confocal microscopy (LSM 880, Zeiss, Germany). The bone mineral apposition rate (MAR, $\mu \mathrm{m} / \mathrm{d}$ ) was measured and calculated by automatic image analysis system.

\section{Biomechanical Test}

A three-point bending test was performed on the tibia specimens to determine the biomechanical properties by a material testing machine (ELF-3510AT, Bose, Inc., USA) as previously described (33). The bones were positioned horizontally on two supports. As the location of the bone repair area, the center of the metaphysis was positioned downward. The load and the displacement of the loading device were collected during each experiment until fracture. Data was recorded to the material testing instrument from the load-deformation curve. The maximum force at failure values $(\mathrm{N})$ were recorded from the load data, and the stiffness $(\mathrm{N} / \mathrm{mm})$ was calculated as the slope of the initial linear uploading portion of the curves.

\section{Statistical Analysis}

SPSS software version 25.0 was used for all statistical analyses. Data were analyzed by two-tailed Student's $t$-test or analysis of variance (ANOVA), with repeated measures where applicable. Differences were determined to be statistically significant when $P$-value $<0.05$, with the data reported as mean \pm SEM.

\section{RESULTS}

\section{Melatonin Promoted the Osteogenesis of BMSCs In Vitro}

First, a CCK-8 assay was conducted to observe whether melatonin can affect the proliferation of BMSCs. As shown in Figure 1A, melatonin promoted cell proliferation, and its effect was not in a dose-dependent manner. The most effective concentration was $100 \mathrm{nM}$, followed by $1 \mu \mathrm{M}, 10 \mathrm{nM}$, and $10 \mu \mathrm{M}$. Then, ALP staining and ARS staining were performed to assess the proosteogenic effect of melatonin in vitro. BMSCs treated with melatonin indicated a higher level of ALP activities compared to the control group (Figures 1B, C). The most effective concentration was also $100 \mathrm{nM}$, followed by $1 \mu \mathrm{M}$ and $10 \mathrm{nM}$, which was consistent with the CCK-8 assay. In addition, ARS staining validated the result. After 14 days of osteogenic induction,
ARS staining revealed an increase in the stained area and extracellular deposition of calcium in the melatonin treatment groups compared with the control group (Figures 1D, E).

To further investigate how melatonin promotes the osteogenesis of BMSCs, we measured the mRNA and protein expressions of osteogenesis-related genes in cultured BMSCs at 3 and 7 days after various gradient concentrations of melatonin treatment. It showed that the mRNA expression levels of osteogenesis-related markers, including ALP, OCN, RUNX2, and osterix were all significantly increased in the melatonin treatment groups compared with the control group (Figure 1F). The most effective concentration is $100 \mathrm{nM}$. Consistently, the protein expression levels of ALP, OCN, and RUNX2 were all significantly increased in the melatonin treatment groups compared with the control group (Figures 1G, H). Immunofluorescence staining also indicated higher expression levels of ALP and OCN after melatonin treatment (Figures 1I, J). Thus, all these data indicated that melatonin promoted the osteogenesis of BMSCs in vitro.

\section{Melatonin Promoted Angiogenesis In Vitro}

Then, the mRNA expressions of angiogenesis-related genes in cultured BMSCs at 3 days were detected after various concentrations of melatonin treatment. The mRNA expression levels of angiogenesis-related markers, including VEGF, angiopoietin-2, and angiopoietin-2, were all significantly upregulated compared with the control group (Figure 2A). After 7 days of melatonin treatment, the protein expression level of VEGF was significantly increased compared with the control group (Figure 2B). Consistently, ELISA and immunofluorescence staining for VEGF both showed significantly higher levels of VEGF in the melatonin-treated groups (Figures 2C, D), Notably, the most effective concentration for all of these assays is also $100 \mathrm{nM}$. It suggested that the most effective concentration of melatonin for promoting osteogenesis and angiogenesis was consistent. These findings imply that melatonin can also promote angiogenesis in vitro.

\section{Melatonin Promoted Osteogenesis- Angiogenesis Coupling In Vitro}

To further assess the angiogenic capability of melatonin, BMSCs were treated with or without $100 \mathrm{nM}$ melatonin, and the conditioned mediums were harvested after 7 days of osteogenic induction. The fresh mediums and the conditioned mediums were used for the following assays, respectively. Scratch wound assay and transwell migration assay were used to explore whether melatonin could affect cell migration. We found that no significant difference in cell migration was observed between the melatonin-treated group and the control group when HUVECs were cultured in fresh medium $(P>0.05)$. However, the migration of the melatonintreated group was incredibly increased compared to the untreated group when HUVECs were cultured in conditioned medium (Figures 3A-D). Consistently, there was no significant difference in the ability to induce capillary tube formation between the melatonin-treated group and the untreated group when HUVECs were cultured in fresh medium $(P>0.05)$. The ability of the melatonin-treated group to induce capillary tube formation was 
A

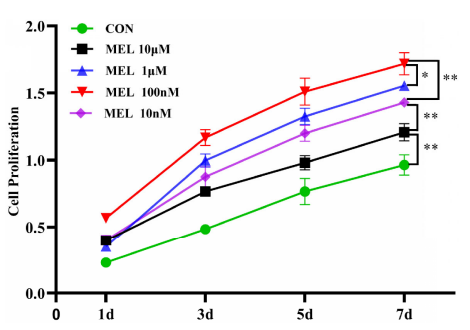

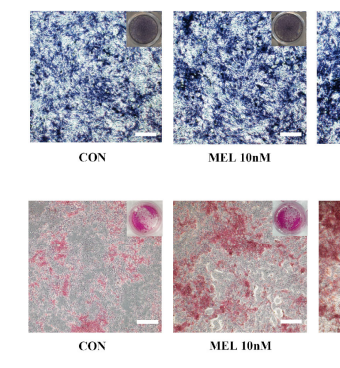
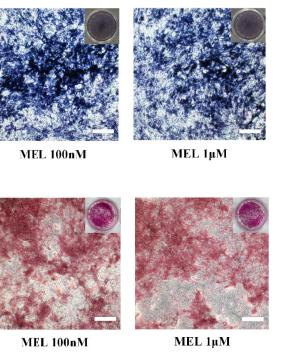

C

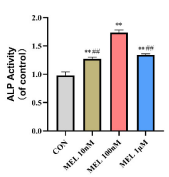

E

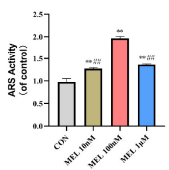

F

G
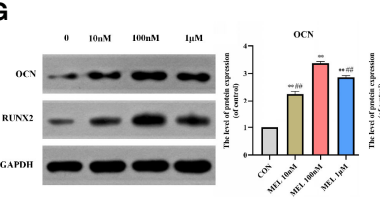

H

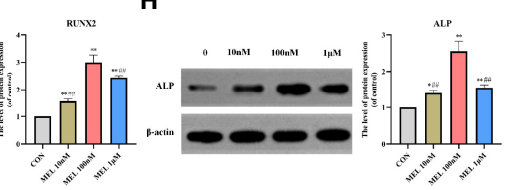

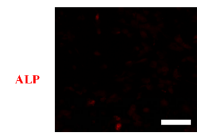

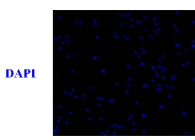

Merge

cos
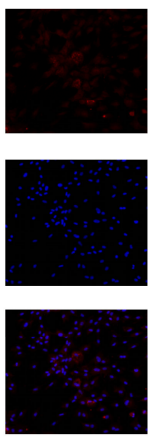

MEL $10 \mathrm{nM}$
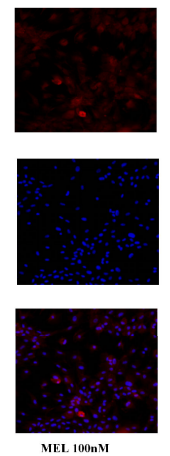
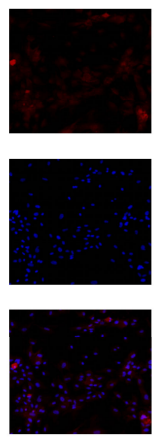

MEL 1MM
J
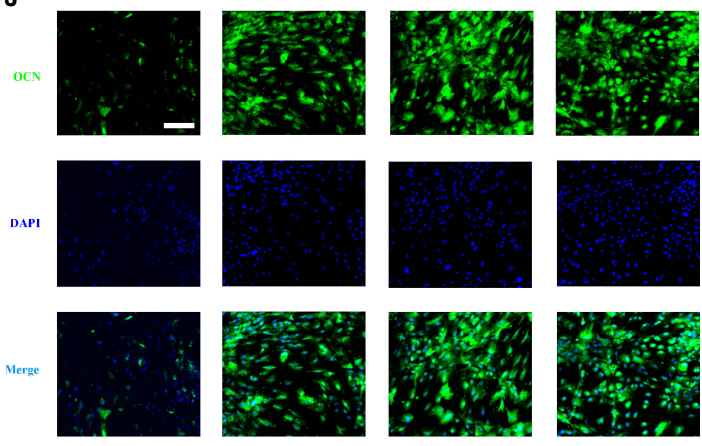

$\operatorname{CON}$

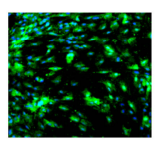

MEL 10nM

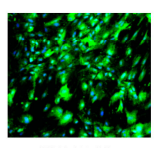

MEL 100nM
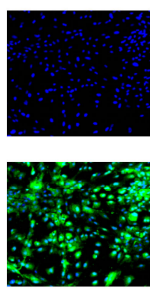

MEL 1 MM

FIGURE 1 | Melatonin promoted the osteogenesis of BMSCs in vitro. (A) The effect of melatonin on BMSC proliferation measured by CCK-8 assays. (B, C) Images and quantification of ALP activity after 7 days of osteogenic induction (scale bars, $200 \mu \mathrm{m}$ ). (D, E) Calcium mineralization was assessed via ARS staining and quantification (scale bars, $200 \mu \mathrm{m}$ ). (F) mRNA expression levels of osteogenesis-related markers in BMSCs following treatment with/without melatonin. (G, H) Protein expression levels of osteogenesis-related markers (OCN, RUNX2, and ALP). (I, J) Immunofluorescent images of BMSCs stained for ALP and OCN (scale bars, 100 $\mu \mathrm{m})$. All the experiments were repeated at least 3 times independently. CCK-8, cell counting kit-8; BMSCs, bone marrow mesenchymal stem cells; ARS, alizarin red $\mathrm{S}$; ALP, alkaline phosphatase; OCN, osteocalcin; RUNX2, runt-related transcription factor 2 . The data are presented as means \pm SEM. ${ }^{*} p<0.05$, ${ }^{*} p<0.01 v s$. control group, ${ }^{\#} p<0.05,{ }^{\# \#} p<0.01$ vs. $100 \mathrm{nM}$ melatonin group.

significantly enhanced compared to the untreated group when HUVECs were cultured in conditioned medium (Figures 3E, F). We also found that the most effective concentration for the abovementioned assays is $100 \mathrm{nM}$, which is consistent with our previous results. The results indicated that melatonin treatment was not able to facilitate HUVEC angiogenesis. However, it implied that melatonin possessed the ability to promote BMSC-mediated angiogenesis. This illustrated that the role of melatonin in promoting angiogenesis is coupled with that in promoting osteogenesis. Collectively, it demonstrated that melatonin promoted osteogenesis-angiogenesis coupling in vitro.

\section{Confirmation of Osteoporosis Model in OVX Rats}

After 3 months of OVX surgery, the efficacy of OVX was confirmed by micro-CT and H\&E staining of tibia bones. 2D images and $3 \mathrm{D}$ vertically sectioned images of the tibia bone were performed in the Sham group and the OVX group (Figures 4A, B). Both 2D and 3D images showed a significant trabecular bone volume, thickness, and density decrease, with a striking trabecular separation increase, compared to the Sham group. To observe this change more clearly, the $2 \mathrm{D}$ scanned images were constructed into $3 \mathrm{D}$ microstructures for analyses (Figure 4C). It showed significantly decreased BMD, BV/TV, and Tb.N in the OVX group compared with the Sham group (Figure 4D). Furthermore, H\&E staining exhibited that the tibia bone in the OVX group was severely damaged, with increased bone trabecular spacing and broken tibia trabeculae, compared with the Sham group (Figure 4E). All of the above-mentioned data demonstrated that the OVX rat model of osteoporosis was successfully established.

\section{Melatonin Enhanced Bone Repairing Ability by Promoting the Osteogenesis of Tibia Defect in OVX Rats}

After confirmation of osteoporosis, a tibia defect model was established in rats and then treated with melatonin for 4 weeks. At week 2, the CON group remained primarily empty in the $3 \mathrm{D}$ reconstruction images. However, a small amount of mineralized 
A

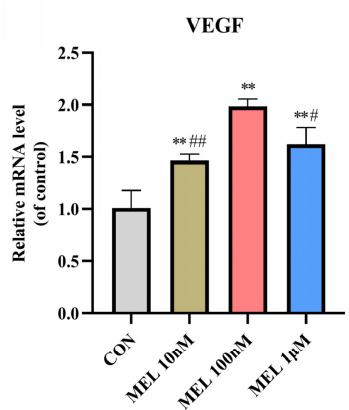

B
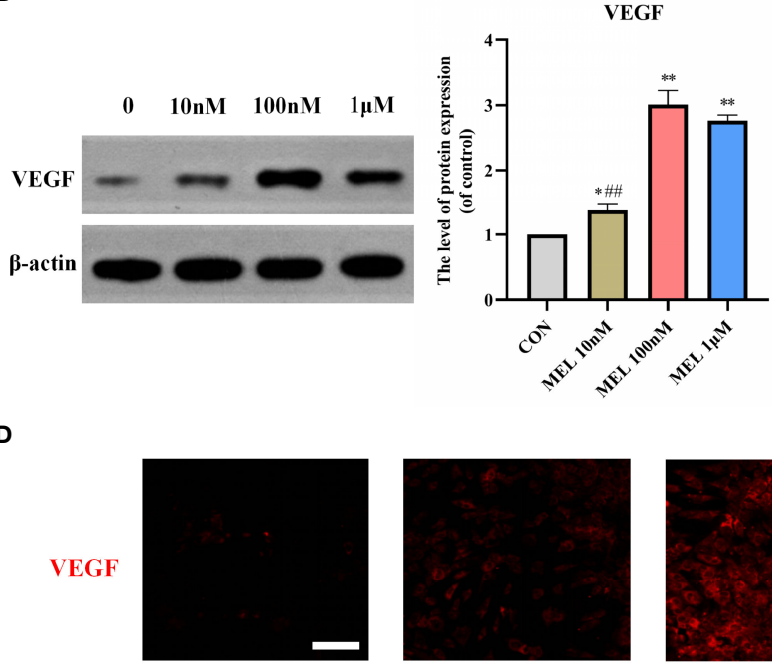

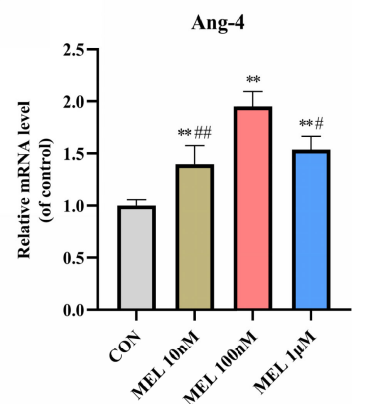

C

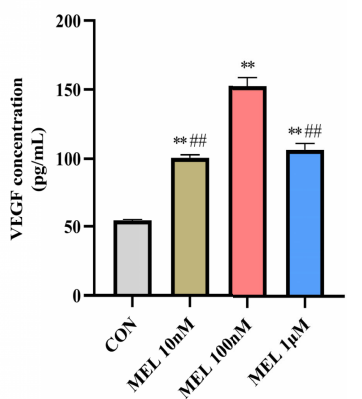

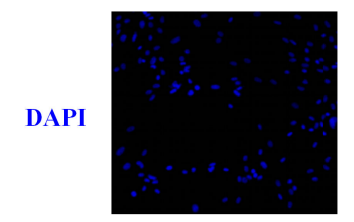
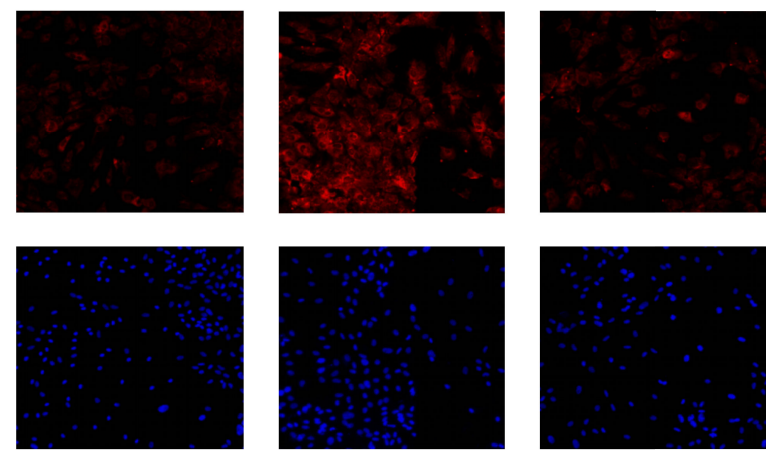

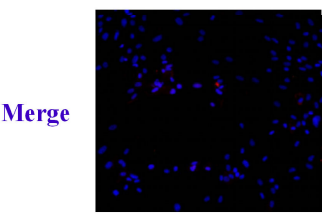

CON

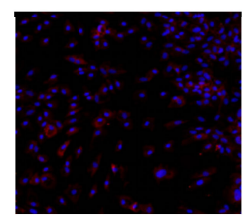

MEL 10nM
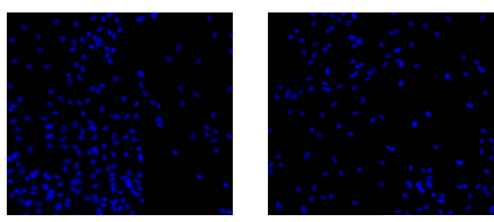

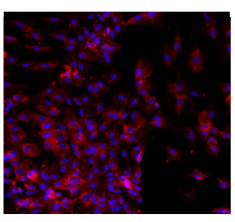

MEL 100nM

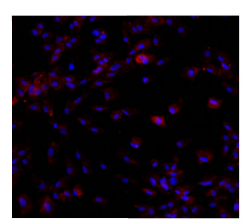

MEL $1 \mu \mathrm{M}$

FIGURE 2 Melatonin promoted angiogenesis in vitro. (A) mRNA expression levels of osteogenesis-related markers in BMSCs following treatment with/without melatonin. (B) Protein expression levels of osteogenesis-related marker VEGF. (C) VEGF content secreted in the supernatant liquid assessed by ELISA kits. (D) Immunofluorescent images of BMSCs stained for VEGF (scale bars, $100 \mu \mathrm{m}$ ). All the experiments were repeated at least 3 times independently. BMSCs, bone marrow mesenchymal stem cells; VEGF, vascular endothelial growth factor. The data are presented as means \pm SEM. ${ }^{*} p<0.05$, ${ }^{\star \star} p<0.01$ vs. control group, ${ }^{\#} p<0.05,{ }^{\# \#} p<0.01$ vs. $100 \mathrm{nM}$ melatonin group.

tissue was predominantly located at the defect periphery in the LMEL and HMEL groups (Figure 5A). At week 4, increased bone volume can be observed in the LMEL and HMEL groups compared with the CON group (Figure 5B). Histological representation of bone formation further confirmed the results of micro-CT (Figures 5C, D). In addition, BMD, BV/TV, Tb.N, and Tb.Sp in the ROI were further analyzed. The CON group showed the lowest values in BMD, BV/TV, and Tb.N, with the highest value in Tb.Sp among all groups at both time points (Figure 5E). It indicated that melatonin treatment promoted the osteogenesis of tibia defect in OVX rats. Meanwhile, Masson's trichrome staining showed that melatonin treatment increased 
A
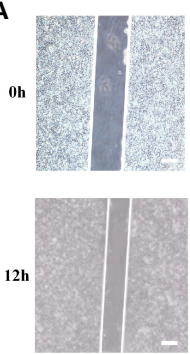

FM-Vehicle

C

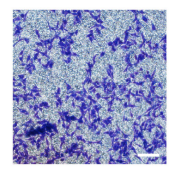

FM-Vehicle

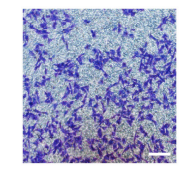

FM+100nM MEL

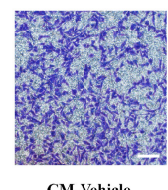

CM-Vehicle

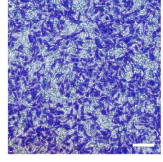

CM+100nM MEL

E

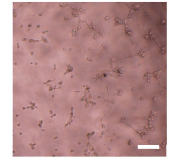

FM-Vehicle

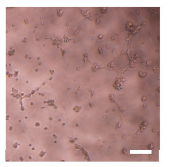

FM+100nM MEL
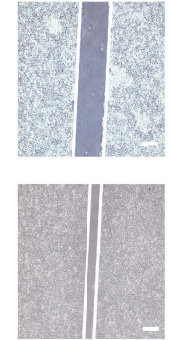

CM-Vehicle

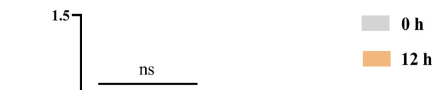

龍

Conditioned Medium $-\quad++\quad--++$

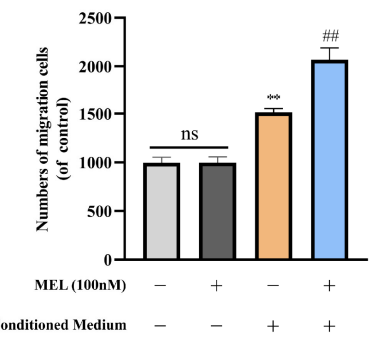

$\mathbf{F}$

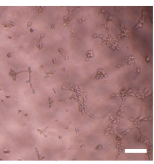

CM-Vehicle

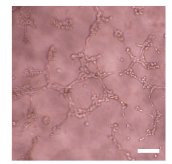

CM+100nM MEL

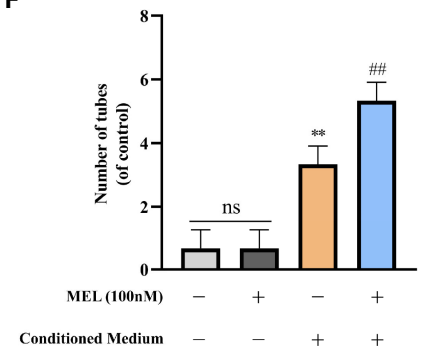

FIGURE 3 | Melatonin promoted osteogenesis-angiogenesis coupling in vitro. (A, B) Scratch wound assay of HUVECs incubated with the indicated mediums (scale bars, $100 \mu \mathrm{m})$. (C, D) Transwell migration assay of HUVECs incubated with the indicated mediums (scale bars, $100 \mu \mathrm{m})$. (E, F) Tube formation assay of HUVECs incubated with the indicated mediums (scale bars, $100 \mu \mathrm{m}$ ). All the experiments were repeated at least 3 times independently. The data are presented as means \pm SEM. ${ }^{* *} p<0.01$ vs. control group, ${ }^{\# \#} p<0.01$ vs. CM-vehicle group. HUVECs, human umbilical vein endothelial cells; FM, fresh medium; CM, conditioned medium. ns, no significance.

the bone mineralization and formation around the tibia defect compared with the CON group (Figures 5F, G). Remarkably, the HMEL group showed a more striking effect than the LMEL group at both time points. This suggested that melatonin therapy may show a dose-dependent manner in vivo. In general, these results revealed that melatonin enhanced the bone repairing ability by promoting the osteogenesis of tibia defect in OVX rats.

\section{Melatonin Accelerated Bone Repair by Promoting the Osteogenesis and Angiogenesis of Tibia Defect in OVX Rats}

Immunohistochemical staining of osteogenesis-related marker (OCN) and angiogenesis-related markers (VEGF and CD31) was further performed. Compared with the CON group, OCN immunostaining was denser and more widely distributed in sections in the melatonin-treated groups (Figure 6A), which was consistent with the results of VEGF and CD31 (Figures 6B, C). The HMEL group showed a more striking effect than the LMEL group at both time points. Sequential fluorescent labeling showed that the distance strip in the melatonin-treated groups was wider than that in the CON group (Figure 6D). The bone mineral deposition rate was analyzed to investigate the bone formation activity. It showed that MAR was significantly improved in the melatonin-treated groups, and this effect was more significant in the HMEL group than that in the LMEL group (Figure 6E). The three-point bending test revealed that the melatonin treatment increased the ultimate load and stiffness compared with the CON group (Figures 6F, G). All these data implied that melatonin could accelerate bone repair and increase bone strength by promoting the osteogenesis and angiogenesis of tibia defect in OVX rats.

\section{DISCUSSION}

The repair of bone defects requires recapitulation of complex signaling cascades, including a series of spatiotemporal angiogenesis and osteogenesis $(34,35)$. However, for 
A

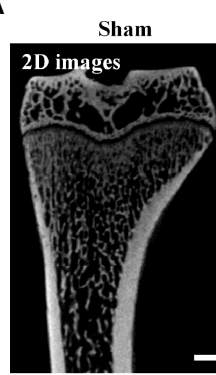

c

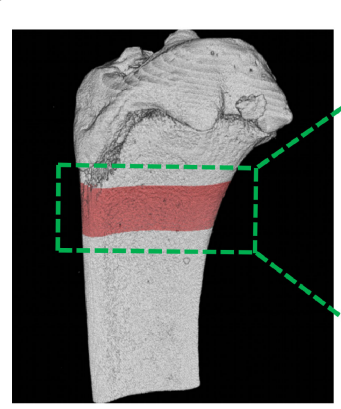

D

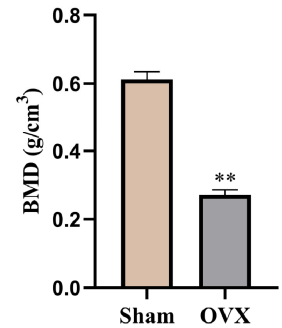

E
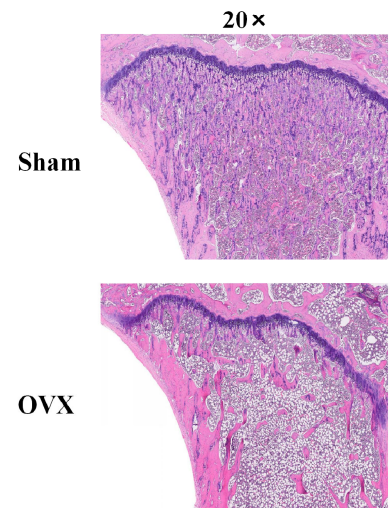

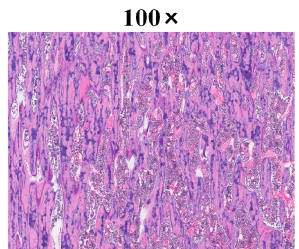

B

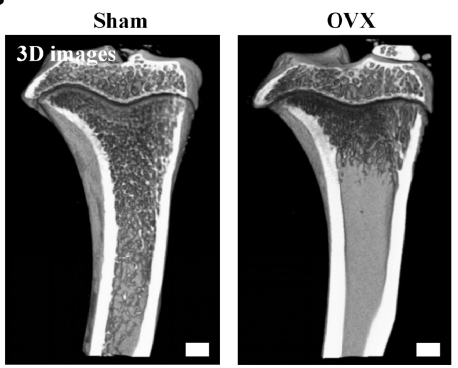

Sham
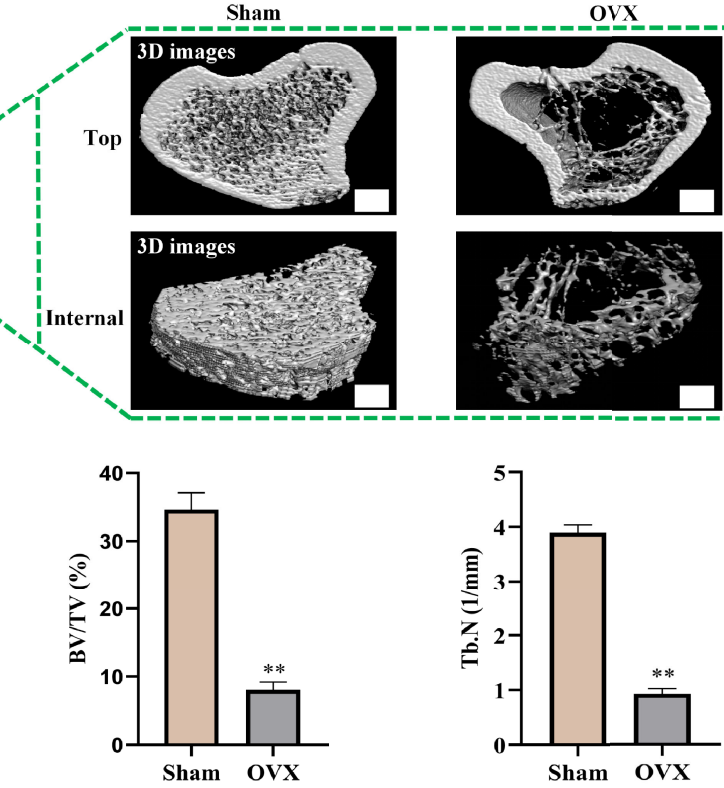

Sham OVX
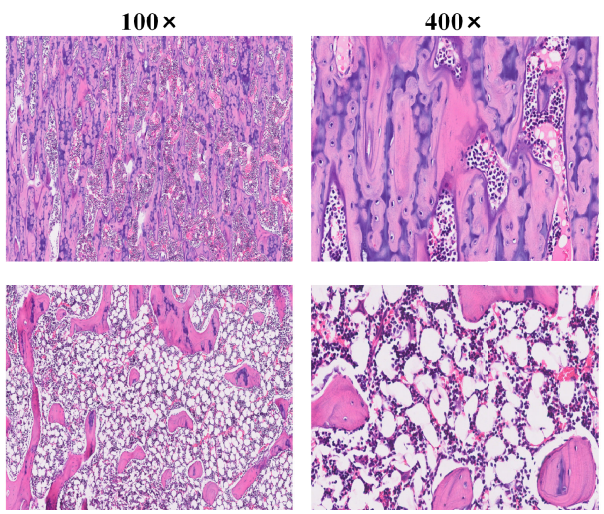

FIGURE 4 | Confirmation of osteoporosis model in ovariectomized rats. (A) 2D images of the tibia bone in two groups (scale bars, 1 mm). (B) Tibia bone visualized and vertical-sectioned images (scale bars, $1 \mathrm{~mm}$ ). (C) 3D constructed images of the tibia proximal metaphysis (top, trabecular bone with cortical bone; internal, trabecular portion) (scale bars, $1 \mathrm{~mm}$ ). (D) Quantitative presentation of microarchitectural parameters including BMD, BV/TV, and Tb.N. (E) H\&E staining images of tibia bone. BMD, bone mineral density; BV/TV, trabecular bone volume; Tb.N, trabecular number. The data are presented as means \pm SEM. ${ }^{\star \star} p<0.01$ vs. Sham group.

osteoporosis, the bone resorption rate is greater than that of new bone formation, with a decreased ability for new bone formation. The clinical therapy of osteoporotic bone defect is more difficult than that of normal bone defect (36). However, conventional treatment option has limited efficacy and is not satisfactory.
Therefore, novel therapeutic drug strategies to tackle osteoporosis and its related complications are warranted, which should be effective, safe, and available. To the best of our knowledge, our study is the first to demonstrate that melatonin could promote osteogenesis-angiogenesis coupling 


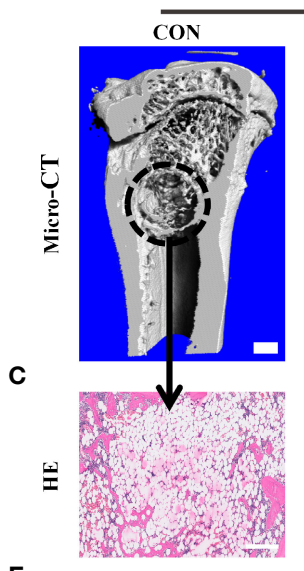

E

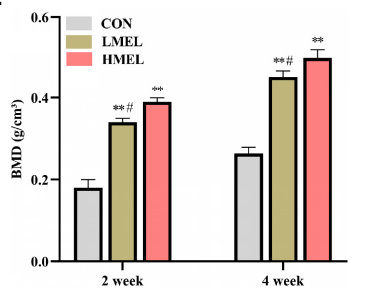

2 week
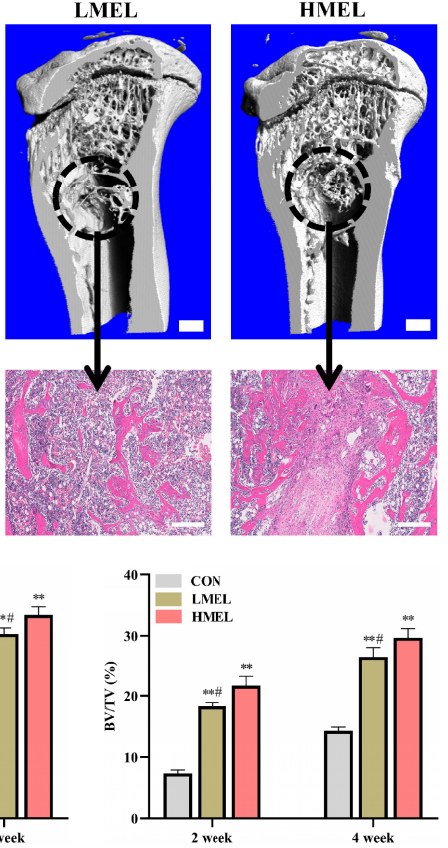

2 week

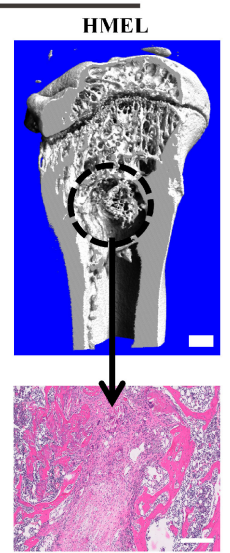

B
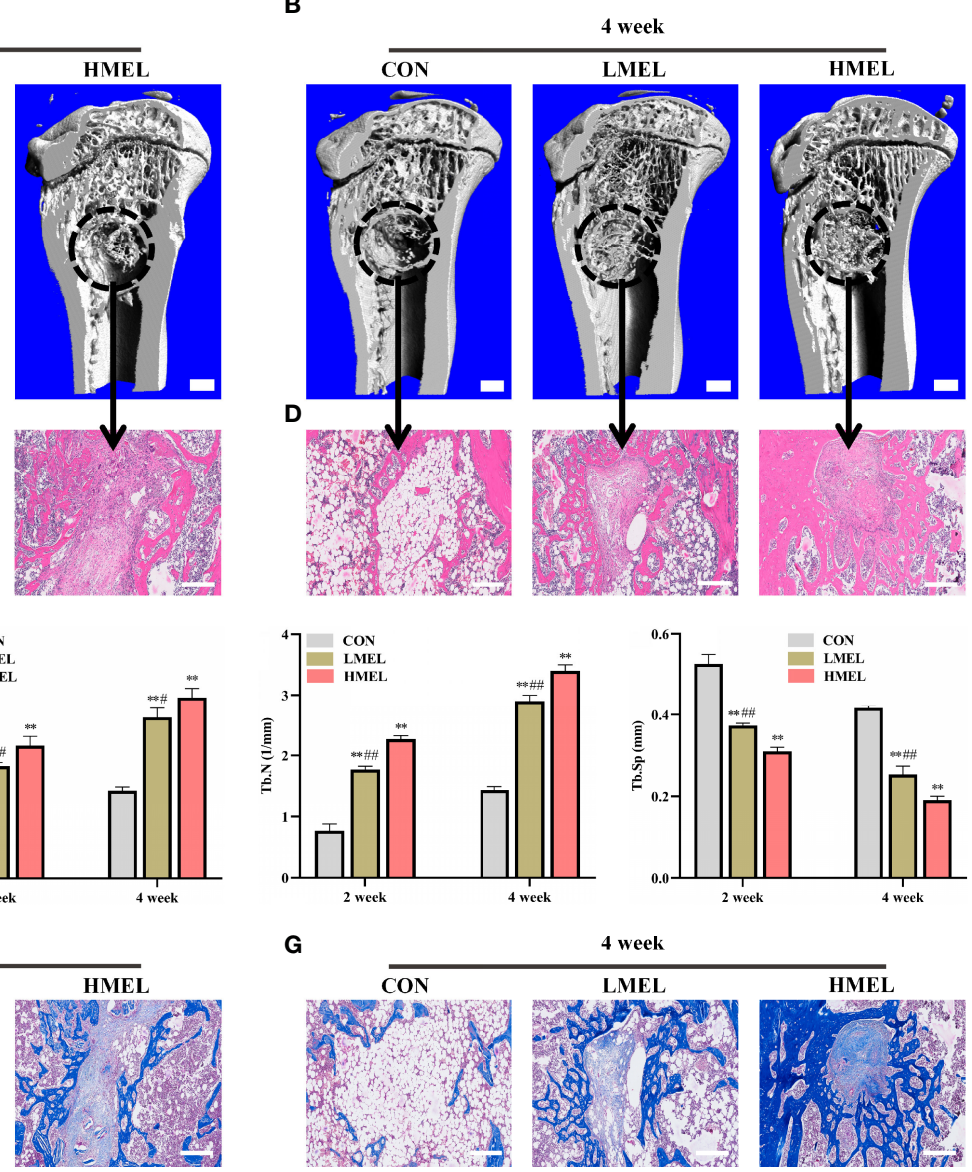

G

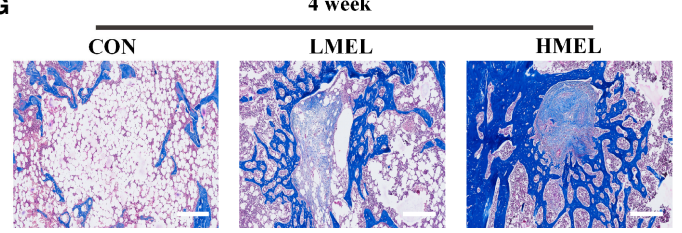

FIGURE 5 | Melatonin enhanced the bone repairing ability by promoting osteogenesis of tibia defect in ovariectomized rats. (A, B) 3D images of mineralized bone formation in tibia defect (scale bars, $1 \mathrm{~mm}$ ). (C, D) Histological assessment of the defect area by H\&E staining (scale bars, $200 \mu \mathrm{m})$. (E) Quantitative presentation of microarchitectural parameters, including BMD, BV/TV, Tb.N, and Tb.Sp. (F, G) Histological assessment of the defect area by Masson's trichrome staining (scale bars, $200 \mu \mathrm{m})$. BMD, bone mineral density, BV/TV, trabecular bone volume, Tb.N, trabecular number; Tb.Sp, trabecular separation; HMEL, high-dose melatonin treatment group. The data are presented as means \pm SEM. ${ }^{\star *} p<0.01$ vs. control group, ${ }^{*} p<0.05$, ${ }^{\# \#} p<0.01$ vs. HMEL group.

in vitro. Meanwhile, we further confirmed that melatonin treatment could accelerate bone repair and increase bone strength by promoting the osteogenesis and angiogenesis of tibia defect in OVX rats.

Bone regeneration is inseparable from the supply of nutrients, and angiogenesis in bone is crucial for bone defect repair (37). Vascularization is the premise of bone defect repair (38), which is a key link in the process of fracture healing and bone defect repair (39). The conventional view is that the relationship between osteogenesis and angiogenesis is one way, mainly manifested as angiogenesis providing essential nutrients for bone regeneration and repair and eliminating metabolic wastes (40). However, recent studies have shown that bone regeneration also plays a vital role in the regeneration of blood vessels within the bone $(41,42)$. Thus, it is critical to focus on the regeneration of blood vessels while investigating bone regeneration.

Increasing studies suggested that melatonin plays beneficial roles in bone metabolism, including bone anabolism as well as anti-bone resorption (43-45). Currently, various studies have been focused on the association between melatonin and osteogenesis. However, few studies are about the effects of melatonin on angiogenesis. In our study, we found that melatonin can promote osteogenesis and angiogenesis simultaneously. An in vitro study showed that melatonin promoted osteogenesis at the same optimal concentration as it promoted angiogenesis. The most effective concentration of both was $100 \mathrm{nM}$. However, it is worth noting that its effect was not in a dose-dependent manner. It indicates that it is important to find the optimal concentration, rather than simply increasing it, for melatonin to maximize its role in osteogenesis and angiogenesis. We further found that melatonin possessed the ability to promote BMSC-mediated angiogenesis and osteogenesisangiogenesis coupling in vitro. When HUVECs were cultured in fresh medium, we found that no significant difference in cell migration and tube formation was observed between the melatonin-treated group and the control group. However, when HUVECs were cultured in conditioned medium, the ability of migration and induction of capillary tube formation 
A

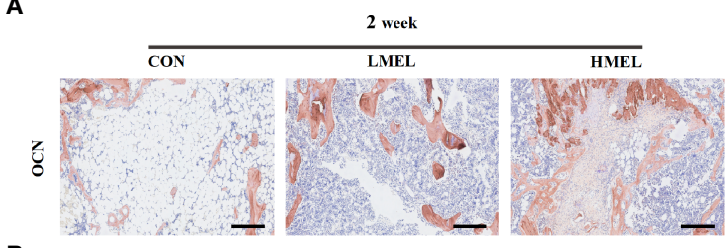

B

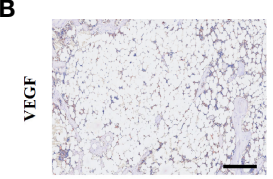

C
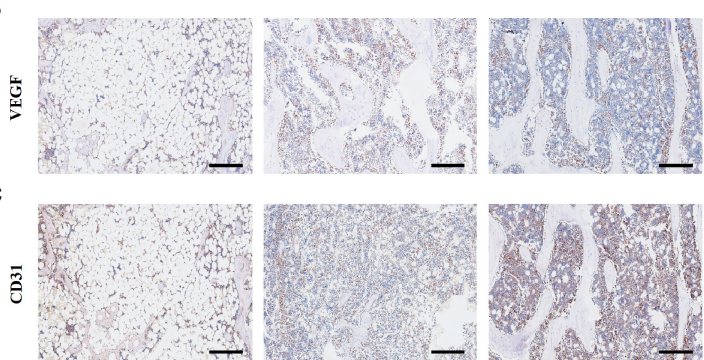

D
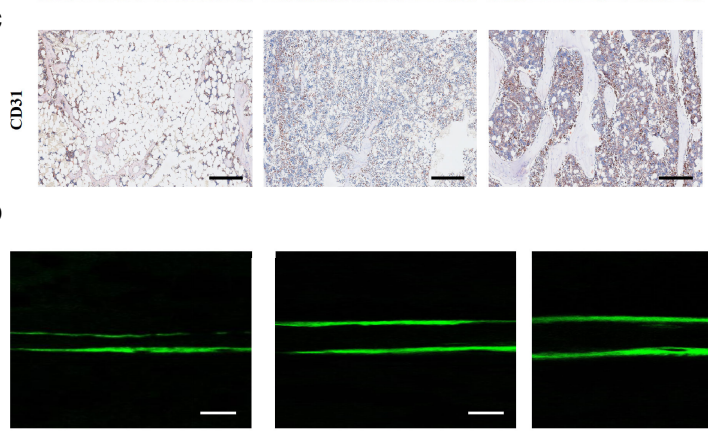

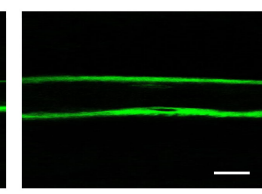

FIGURE 6 | Melatonin accelerated bone repair by promoting the osteogenesis and angiogenesis of tibia defect in ovariectomized rats. (A) Images of immunohistochemical staining of osteocalcin in the tibia defect (scale bars, $200 \mu \mathrm{m}$ ). (B) Images of immunohistochemical staining of vascular endothelial growth factor in the tibia defect (scale bars, $200 \mu \mathrm{m}$ ). (C) Images of immunohistochemical staining of CD31 in the tibia defect (scale bars, $200 \mu \mathrm{m})$. (D) New bone formation was detected by sequential fluorescent labeling of calcein (scale bars, $10 \mu \mathrm{m}$ ). (E) Quantitative analysis of mineral apposition rate. (F) Maximum force determined experimentally by three-point bending test. (G) Stiffness determined experimentally by three-point bending test. The data are presented as means \pm SEM. ${ }^{*} p<0.05$, ${ }^{* \star} p<0.01$ vs. control group, ${ }^{\#} p<0.05$ vs. high-dose melatonin treatment group.

of the melatonin-treated group was significantly enhanced compared to the untreated group. The communication between vascular endothelial cells and BMSCs was significantly critical in bone remodeling. The process can be amplified by multiple elements, which participated in the recruitment, differentiation, and proliferation of vascular endothelial cells and BMSCs (46). This association has been illustrated to be due to an osteoblastic and angiogenic factor (VEGF), which was consistent with our findings (47). Combined with the results of the VEGF-related assays in this study, it suggested that melatonin could promote BMSC-mediated angiogenesis by upregulating the VEGF levels.

Previous studies, including animal experiments and clinical findings, have shown that melatonin has a notably antiosteoporosis effect and high safety profile (48-52). Amstrup et al. demonstrated that melatonin could improve BMD at the femoral neck in postmenopausal women with osteopenia (53). A randomized controlled trial suggested that melatonin treatment is safe in postmenopausal women with osteopenia, and small doses of melatonin can improve sleep quality (54). However, the studies on melatonin mostly focused on the effects of melatonin therapy on osteoporosis. Relative mechanisms are mainly focused on the fact that melatonin can promote osteogenesis in osteoblasts (55-57) and inhibit osteolysis in osteoclasts (58-60).
There is little evidence about exploring the relationship between melatonin and angiogenesis. Ramírez-Fernández et al. showed that melatonin could promote angiogenesis in a bone defect rabbit model and may have potential beneficial effects on bone defect repair (26). Hu et al. demonstrated that melatonin could protect cortical bone-derived stem cells against $\gamma$-ray radiation and assist in the healing of postradiation bone defects (61). Yildirimturk et al. found that melatonin showed beneficial effects on the healing of bone defects in streptozotocin-induced diabetic rats (62). However, these studies lacked further clarify how melatonin affects angiogenesis and explore its effects on osteoporotic bone defect. Our study is the first to demonstrated that melatonin could promote BMSCs-mediated angiogenesis by upregulating VEGF levels and promote osteogenesis-angiogenesis coupling in vitro. In addition, our study is novel in showing that melatonin could accelerate bone repair by promoting osteogenesis and angiogenesis of tibia defect in OVX rats. Therefore, our study is the first to indicate that melatonin treatment was able to accelerate bone repair in rats with osteoporotic bone defect, which was potentially effective to bone regeneration. However, the limitation of our study is that the exact pathway underlying melatonin promoting osteogenesis-angiogenesis coupling has not been clarified, which is our ongoing research. 


\section{CONCLUSION}

In conclusion, our results demonstrated that melatonin could accelerate osteoporotic bone repair by promoting osteogenesisangiogenesis coupling. Further investigation is undertaken about the underlying mechanism about how the osteogenesisangiogenesis coupling process is promoted by melatonin. These findings can advance our thinking about that the application of melatonin may provide new insight and strategy for bone regeneration, and hence it could be a promising therapeutic remedy against osteoporosis and osteoporotic bone defect.

\section{DATA AVAILABILITY STATEMENT}

The original contributions presented in the study are included in the article/supplementary material. Further inquiries can be directed to the corresponding author.

\section{ETHICS STATEMENT}

The animal study was reviewed and approved by the Animal Care and Ethics Committee of the Southern Medical University.

\section{REFERENCES}

1. Panday K, Gona A, Humphrey MB. Medication-Induced Osteoporosis: Screening and Treatment Strategies. Ther Adv Musculoskelet Dis (2014) 6 (5):185-202. doi: 10.1177/1759720X14546350

2. Ghosh M, Majumdar SR. Antihypertensive Medications, Bone Mineral Density, and Fractures: A Review of Old Cardiac Drugs That Provides New Insights Into Osteoporosis. Endocrine (2014) 46(3):397-405. doi: 10.1007/ s12020-014-0167-4

3. Rosen CJ. The Epidemiology and Pathogenesis of Osteoporosis. (2020). Available at: www.endotext.org.

4. Sözen T, Özışık L, Başaran NÇ. An Overview and Management of Osteoporosis. Eur J Rheumatol (2017) 4(1):46-56. doi: 10.5152/ eurjrheum.2016.048

5. Keller J, Catala-Lehnen P, Huebner AK, Jeschke A, Heckt T, Lueth A, et al. Calcitonin Controls Bone Formation by Inhibiting the Release of Sphingosine 1-Phosphate From Osteoclasts. Nat Commun (2014) 5:5215. doi: 10.1038/ ncomms6215

6. Namkung-Matthai H, Appleyard R, Jansen J, Hao LJ, Maastricht S, Swain M, et al. Osteoporosis Influences the Early Period of Fracture Healing in a Rat Osteoporotic Model. Bone (2001) 28(1):80-6. doi: 10.1016/s8756-3282(00) 00414-2

7. Hegde V, Jo JE, Andreopoulou P, Lane JM. Effect of Osteoporosis Medications on Fracture Healing. Osteoporos Int (2016) 27(3):861-71. doi: 10.1007/s00198-015-3331-7

8. McCann RM, Colleary G, Geddis C, Clarke SA, Jordan GR, Dickson GR, et al. Effect of Osteoporosis on Bone Mineral Density and Fracture Repair in a Rat Femoral Fracture Model. J Orthop Res (2008) 26(3):384-93. doi: 10.1002/ jor.20505

9. Hao YJ, Zhang G, Wang YS, Qin L, Hung WY, Leung K, et al. Changes of Microstructure and Mineralized Tissue in the Middle and Late Phase of Osteoporotic Fracture Healing in Rats. Bone (2007) 41(4):631-8. doi: 10.1016/ j.bone.2007.06.006

10. Hak DJ. The Biology of Fracture Healing in Osteoporosis and in the Presence of Anti-Osteoporotic Drugs. Injury (2018) 49(8):1461-5. doi: 10.1016/ j.injury.2018.04.016

\section{AUTHOR CONTRIBUTIONS}

YL conceived and designed the experiments. SZ, CZ, and HY carried out the experiments. JL, ZF, and LL analyzed the data. All authors were involved in writing the paper and had final approval of the submitted and published versions.

\section{FUNDING}

This study was sponsored by the National Natural Science Foundation of China (no. 81674095), the National Administration of Traditional Chinese Medicine TCM Inheritance and Innovation "Hundred-Thousand-Ten Thousand" Talents Project (QiHuang Scholar)-National TCM Leading Personnel Support Program (NATCM Personnel and Education Department, no. F119090038), the Innovation Team and Talents Cultivation Program of National Administration of Traditional Chinese Medicine (no. ZYYCXTD-C-202003), and the Sanming Project of Medicine in Shenzhen (no. SZZYSM202108013). The funders had no role in the study design, data collection, and analysis, or preparation of the manuscript.

11. Ding WG, Wei ZX, Liu JB. Reduced Local Blood Supply to the Tibial Metaphysis Is Associated With Ovariectomy-Induced Osteoporosis in Mice. Connect Tissue Res (2011) 52(1):25-9. doi: 10.3109/03008201003783011

12. Wang JW, Li W, Xu SW, Yang DS, Wang Y, Lin M, et al. Osteoporosis Influences the Middle and Late Periods of Fracture Healing in a Rat Osteoporotic Model. Chin J Traumatol (2005) 8(2):111-6.

13. Lin K, Xia L, Li H, Jiang X, Pan H, Xu Y, et al. Enhanced Osteoporotic Bone Regeneration by Strontium-Substituted Calcium Silicate Bioactive Ceramics. Biomaterials (2013) 34(38):10028-42. doi: 10.1016/j.biomaterials.2013.09.056

14. Stehle JH, Saade A, Rawashdeh O, Ackermann K, Jilg A, Sebestény T, et al. A Survey of Molecular Details in the Human Pineal Gland in the Light of Phylogeny, Structure, Function and Chronobiological Diseases. J Pineal Res (2011) 51(1):17-43. doi: 10.1111/j.1600-079X.2011.00856.x

15. Dubocovich ML, Markowska M. Functional MT1 and MT2 Melatonin Receptors in Mammals. Endocrine (2005) 27(2):101-10. doi: 10.1385/ ENDO:27:2:101

16. Tan DX, Manchester LC, Reiter RJ, Qi WB, Zhang M, Weintraub ST, et al. Identification of Highly Elevated Levels of Melatonin in Bone Marrow: Its Origin and Significance. Biochim Biophys Acta (1999) 1472(1-2):206-14. doi: 10.1016/s0304-4165(99)00125-7

17. Slominski RM, Reiter RJ, Schlabritz-Loutsevitch N, Ostrom RS, Slominski AT. Melatonin Membrane Receptors in Peripheral Tissues: Distribution and Functions. Mol Cell Endocrinol (2012) 351(2):152-66. doi: 10.1016/ j.mce.2012.01.004

18. Amstrup AK, Sikjaer T, Mosekilde L, Rejnmark L. Melatonin and the Skeleton. Osteoporos Int (2013) 24(12):2919-27. doi: 10.1007/s00198-0132404-8

19. de Villiers TJ, Chines AA, Palacios S, Lips P, Sawicki AZ, Levine AB, et al. Safety and Tolerability of Bazedoxifene in Postmenopausal Women With Osteoporosis: Results of a 5-Year, Randomized, Placebo-Controlled Phase 3 Trial. Osteoporos Int (2011) 22(2):567-76. doi: 10.1007/s00198-010-1302-6

20. Maria S, Samsonraj RM, Munmun F, Glas J, Silvestros M, Kotlarczyk MP, et al. Biological Effects of Melatonin on Osteoblast/Osteoclast Cocultures, Bone, and Quality of Life: Implications of a Role for MT2 Melatonin Receptors, MEK1/2, and MEK5 in Melatonin-Mediated Osteoblastogenesis. J Pineal Res (2018) 64(3):1-12. doi: 10.1111/jpi.12465 
21. Park KH, Kang JW, Lee EM, Kim JS, Rhee YH, Kim M, et al. Melatonin Promotes Osteoblastic Differentiation Through the BMP/ERK/Wnt Signaling Pathways. J Pineal Res (2011) 51(2):187-94. doi: 10.1111/j.1600079X.2011.00875.x

22. Zhu G, Ma B, Dong P, Shang J, Gu X, Zi Y. Melatonin Promotes Osteoblastic Differentiation and Regulates PDGF/AKT Signaling Pathway. Cell Biol Int (2020) 44(2):402-11. doi: 10.1002/cbin.11240

23. Zhang J, Jia G, Xue P, Li Z. Melatonin Restores Osteoporosis-Impaired Osteogenic Potential of Bone Marrow Mesenchymal Stem Cells and Alleviates Bone Loss Through the HGF/PTEN/Wnt/B-Catenin Axis. Ther Adv Chronic Dis (2021) 12:2040622321995685. doi: 10.1177/2040622321995685

24. Dong P, Gu X, Zhu G, Li M, Ma B, Zi Y. Melatonin Induces Osteoblastic Differentiation of Mesenchymal Stem Cells and Promotes Fracture Healing in a Rat Model of Femoral Fracture via Neuropeptide Y/Neuropeptide Y Receptor Y1 Signaling. Pharmacology (2018) 102(5-6):272-80. doi: 10.1159/ 000492576

25. Grellier M, Granja PL, Fricain JC, Bidarra SJ, Renard M, Bareille R, et al. The Effect of the Co-Immobilization of Human Osteoprogenitors and Endothelial Cells Within Alginate Microspheres on Mineralization in a Bone Defect. Biomaterials (2009) 30(19):3271-8. doi: 10.1016/j.biomaterials.2009.02.033

26. Ramírez-Fernández MP, Calvo-Guirado JL, De-Val JE, Delgado-Ruiz RA, Negri B, Pardo-Zamora G, et al. Melatonin Promotes Angiogenesis During Repair of Bone Defects: A Radiological and Histomorphometric Study in Rabbit Tibiae. Clin Oral Investig (2013) 17(1):147-58. doi: 10.1007/s00784012-0684-6

27. Qin G, Li Y, Wang H, Yang J, Chen Q, Tang H, et al. Lysine-Specific Demethylase 4a Regulates Osteogenic Differentiation via Regulating the Binding Ability of H3K9me3 With the Promoters of Runx2, Osterix and Osteocalcin. J Biomed Nanotechnol (2020) 16(6):899-909. doi: 10.1166/ jbn.2020.2929

28. Zeng Q, Zhou Y, Liang D, He H, Liu X, Zhu R, et al. Exosomes Secreted From Bone Marrow Mesenchymal Stem Cells Attenuate Oxygen-Glucose Deprivation/Reoxygenation-Induced Pyroptosis in PC12 Cells by Promoting AMPK-Dependent Autophagic Flux. Front Cell Neurosci (2020) 14:182. doi: $10.3389 /$ fncel.2020.00182

29. Kharode YP, Sharp MC, Bodine PV. Utility of the Ovariectomized Rat as a Model for Human Osteoporosis in Drug Discovery. Methods Mol Biol (2008) 455:111-24. doi: 10.1007/978-1-59745-104-8_8

30. Zhou W, Liu Y, Shen J, Yu B, Bai J, Lin J, et al. Melatonin Increases Bone Mass Around the Prostheses of OVX Rats by Ameliorating Mitochondrial Oxidative Stress via the SIRT3/SOD2 Signaling Pathway. Oxid Med Cell Longev (2019) 2019:4019619. doi: 10.1155/2019/4019619

31. Chen W, Chen X, Chen AC, Shi Q, Pan G, Pei M, et al. Melatonin Restores the Osteoporosis-Impaired Osteogenic Potential of Bone Marrow Mesenchymal Stem Cells by Preserving SIRT1-Mediated Intracellular Antioxidant Properties. Free Radic Biol Med (2020) 146:92-106. doi: 10.1016/ j.freeradbiomed.2019.10.412

32. Tao ZS, Lu HL, Ma NF, Zhang RT, Li Y, Yang M, et al. Rapamycin Could Increase the Effects of Melatonin Against Age-Dependent Bone Loss. $Z$ Gerontol Geriatr (2020) 53(7):671-8. doi: 10.1007/s00391-019-01659-4

33. Osuna L, Soares CJ, Vilela A, Irie MS, Versluis A, Soares P. Influence of Bone Defect Position and Span in 3-Point Bending Tests: Experimental and Finite Element Analysis. Braz Oral Res (2020) 35:e001. doi: 10.1590/1807-3107bor2021.vol35.0001

34. Mousaei GM, Matin MM, Kazemi MH, Naderi-Meshkin H, Moradi A, Rajabioun M, et al. Application of Mesenchymal Stem Cells to Enhance Non-Union Bone Fracture Healing. J Biomed Mater Res A (2019) 107(2):30111. doi: $10.1002 / j b m . a .36441$

35. Ye D, Chen C, Wang Q, Zhang Q, Li S, Liu H. Short-Wave Enhances Mesenchymal Stem Cell Recruitment in Fracture Healing by Increasing HIF-1 in Callus. Stem Cell Res Ther (2020) 11(1):382. doi: 10.1186/s13287020-01888-0

36. Zhang Y, Cheng N, Miron R, Shi B, Cheng X. Delivery of PDGF-B and BMP-7 by Mesoporous Bioglass/Silk Fibrin Scaffolds for the Repair of Osteoporotic Defects. Biomaterials (2012) 33(28):6698-708. doi: 10.1016/j.biomaterials. 2012.06.021

37. Jin L, Li P, Wang YC, Feng L, Xu R, Yang DB, et al. Studies of Superb Microvascular Imaging and Contrast-Enhanced Ultrasonography in the
Evaluation of Vascularization in Early Bone Regeneration. I Ultrasound Med (2019) 38(11):2963-71. doi: 10.1002/jum.15002

38. Linsley CS, Wu BM, Tawil B. Mesenchymal Stem Cell Growth on and Mechanical Properties of Fibrin-Based Biomimetic Bone Scaffolds. J Biomed Mater Res A (2016) 104(12):2945-53. doi: 10.1002/jbm.a.35840

39. Kirkeby OJ, Nordsletten L, Skjeldal S, Berg LT. Circulation in Corticocancellous Bone Grafts Measured With Laser Doppler Flowmetry. Exp Study Rats Scand J Plast Reconstr Surg Handb Surg (1994) 28(4):249-54. doi: 10.3109/02844319409022007

40. Lafage-Proust MH, Prisby R, Roche B, Vico L. Bone Vascularization and Remodeling. Joint Bone Spine (2010) 77(6):521-4. doi: 10.1016/ j.jbspin.2010.09.009

41. Kusumbe AP, Ramasamy SK, Adams RH. Coupling of Angiogenesis and Osteogenesis by a Specific Vessel Subtype in Bone. Nature (2014) 507 (7492):323-8. doi: 10.1038/nature13145

42. Dhandapani R, Krishnan PD, Zennifer A, Kannan V, Manigandan A, Arul $\mathrm{MR}$, et al. Additive Manufacturing of Biodegradable Porous Orthopaedic Screw. Bioact Mater (2020) 5(3):458-67. doi: 10.1016/j.bioactmat.2020.03.009

43. Satomura K, Tobiume S, Tokuyama R, Yamasaki Y, Kudoh K, Maeda E, et al. Melatonin at Pharmacological Doses Enhances Human Osteoblastic Differentiation In Vitro and Promotes Mouse Cortical Bone Formation In Vivo. J Pineal Res (2007) 42(3):231-9. doi: 10.1111/j.1600-079X.2006.00410.x

44. Zhang L, Su P, Xu C, Chen C, Liang A, Du K, et al. Melatonin Inhibits Adipogenesis and Enhances Osteogenesis of Human Mesenchymal Stem Cells by Suppressing Ppary Expression and Enhancing Runx2 Expression. J Pineal Res (2010) 49(4):364-72. doi: 10.1111/j.1600-079X.2010.00803.x

45. Histing T, Anton C, Scheuer C, Garcia P, Holstein JH, Klein M, et al. Melatonin Impairs Fracture Healing by Suppressing RANKL-Mediated Bone Remodeling. J Surg Res (2012) 173(1):83-90. doi: 10.1016/j.jss.2010.08.036

46. Clarkin CE, Emery RJ, Pitsillides AA, Wheeler-Jones CP. Evaluation of VEGFMediated Signaling in Primary Human Cells Reveals a Paracrine Action for VEGF in Osteoblast-Mediated Crosstalk to Endothelial Cells. J Cell Physiol (2008) 214(2):537-44. doi: 10.1002/jcp.21234

47. Leach JK, Kaigler D, Wang Z, Krebsbach PH, Mooney DJ. Coating of VEGFReleasing Scaffolds With Bioactive Glass for Angiogenesis and Bone Regeneration. Biomaterials (2006) 27(17):3249-55. doi: 10.1016/ j.biomaterials.2006.01.033

48. Uslu S, Uysal A, Oktem G, Yurtseven M, Tanyalçin T, Bașdemir G. Constructive Effect of Exogenous Melatonin Against Osteoporosis After Ovariectomy in Rats. Anal Quant Cytol Histol (2007) 29(5):317-25.

49. Zhang WL, Meng HZ, Yang RF, Yang MW, Sun GH, Liu JH, et al. Melatonin Suppresses Autophagy in Type 2 Diabetic Osteoporosis. Oncotarget (2016) 7 (32):52179-94. doi: 10.18632/oncotarget.10538

50. Chu ZM, Li HB, Sun SX, Jiang YC, Wang B, Dong YF. Melatonin Promotes Osteoblast Differentiation of Bone Marrow Mesenchymal Stem Cells in Aged Rats. Eur Rev Med Pharmacol Sci (2017) 21(19):4446-56.

51. Egermann M, Gerhardt C, Barth A, Maestroni GJ, Schneider E, Alini M. Pinealectomy Affects Bone Mineral Density and Structure-An Experimental Study in Sheep. BMC Musculoskelet Disord (2011) 12:271. doi: 10.1186/14712474-12-271

52. Wang X, Liang T, Zhu Y, Qiu J, Qiu X, Lian C, et al. Correction to: Melatonin Prevents Bone Destruction in Mice With Retinoic Acid-Induced Osteoporosis. Mol Med (2021) 27(1):136. doi: 10.1186/s10020-021-00401-4

53. Amstrup AK, Sikjaer T, Heickendorff L, Mosekilde L, Rejnmark L. Melatonin Improves Bone Mineral Density at the Femoral Neck in Postmenopausal Women With Osteopenia: A Randomized Controlled Trial. J Pineal Res (2015) 59(2):221-9. doi: 10.1111/jpi.12252

54. Amstrup AK, Sikjaer T, Mosekilde L, Rejnmark L. The Effect of Melatonin Treatment on Postural Stability, Muscle Strength, and Quality of Life and Sleep in Postmenopausal Women: A Randomized Controlled Trial. Nutr J (2015) 14:102. doi: 10.1186/s12937-015-0093-1

55. Rafat A, Mohammadi RA, Alizadeh A, Hashemi-Firouzi N, Golipoor Z. Comparison of The Melatonin Preconditioning Efficacy Between Bone Marrow and Adipose-Derived Mesenchymal Stem Cells. Cell J (2019) 20 (4):450-8. doi: 10.22074/cellj.2019.5507

56. Satué M, Ramis JM, Del MAM, Monjo M. A New Role for 5Methoxytryptophol on Bone Cells Function In Vitro. J Cell Biochem (2015) 116(4):551-8. doi: 10.1002/jcb.25005 
57. Xiong XC, Zhu Y, Ge R, Liu LF, Yuan W. Effect of Melatonin on the Extracellular-Regulated Kinase Signal Pathway Activation and Human Osteoblastic Cell Line hFOB 1.19 Proliferation. Int J Mol Sci (2015) 16 (5):10337-53. doi: 10.3390/ijms160510337

58. Ikebuchi Y, Aoki S, Honma M, Hayashi M, Sugamori Y, Khan M, et al. Coupling of Bone Resorption and Formation by RANKL Reverse Signalling. Nature (2018) 561(7722):195-200. doi: 10.1038/s41586-018-0482-7

59. Ping Z, Wang Z, Shi J, Wang L, Guo X, Zhou W, et al. Inhibitory Effects of Melatonin on Titanium Particle-Induced Inflammatory Bone Resorption and Osteoclastogenesis via Suppression of NF-kb Signaling. Acta Biomater (2017) 62:362-71. doi: 10.1016/j.actbio.2017.08.046

60. Kim HJ, Kim HJ, Bae MK, Kim YD. Suppression of Osteoclastogenesis by Melatonin: A Melatonin Receptor-Independent Action. Int J Mol Sci (2017) 18 (6):1-13. doi: 10.3390/ijms18061142

61. Hu W, Liang JW, Liao S, Zhao ZD, Wang YX, Mao XF, et al. Melatonin Attenuates Radiation-Induced Cortical Bone-Derived Stem Cells Injury and Enhances Bone Repair in Postradiation Femoral Defect Model. Mil Med Res (2021) 8(1):61. doi: 10.1186/s40779-021-00355-y

62. Yildirimturk S, Batu S, Alatli C, Olgac V, Firat D, Sirin Y. The Effects of Supplemental Melatonin Administration on the Healing of Bone Defects in
Streptozotocin-Induced Diabetic Rats. J Appl Oral Sci (2016) 24(3):239-49. doi: 10.1590/1678-775720150570

Conflict of Interest: The authors declare that the research was conducted in the absence of any commercial or financial relationships that could be construed as a potential conflict of interest.

Publisher's Note: All claims expressed in this article are solely those of the authors and do not necessarily represent those of their affiliated organizations, or those of the publisher, the editors and the reviewers. Any product that may be evaluated in this article, or claim that may be made by its manufacturer, is not guaranteed or endorsed by the publisher.

Copyright $\odot 2022$ Zheng, Zhou, Yang, Li, Feng, Liao and Li. This is an open-access article distributed under the terms of the Creative Commons Attribution License (CC BY). The use, distribution or reproduction in other forums is permitted, provided the original author(s) and the copyright owner(s) are credited and that the original publication in this journal is cited, in accordance with accepted academic practice. No use, distribution or reproduction is permitted which does not comply with these terms. 